\title{
Exoplanet Biosignatures: At the Dawn of a New Era of Planetary Observations
}

\author{
Nancy Y. Kiang,,2,3 Shawn Domagal-Goldman,,3,4 Mary N. Parenteau,3 David C. Catling,3 Yuka Fujii, \\ Victoria S. Meadows, ${ }^{3,8}$ Edward W. Schwieterman, $3,9,10,11$ and Sara I. Walker ${ }^{11,12,13,14}$
}

\begin{abstract}
The rapid rate of discoveries of exoplanets has expanded the scope of the science possible for the remote detection of life beyond Earth. The Exoplanet Biosignatures Workshop Without Walls (EBWWW) held in 2016 engaged the international scientific community across diverse scientific disciplines, to assess the state of the science and technology in the search for life on exoplanets, and to identify paths for progress. The workshop activities resulted in five major review papers, which provide (1) an encyclopedic review of known and proposed biosignatures and models used to ascertain them (Schwieterman et al., 2018 in this issue); (2) an in-depth review of $\mathrm{O}_{2}$ as a biosignature, rigorously examining the nuances of false positives and false negatives for evidence of life (Meadows et al., 2018 in this issue); (3) a Bayesian framework to comprehensively organize current understanding to quantify confidence in biosignature assessments (Catling et al., 2018 in this issue); (4) an extension of that Bayesian framework in anticipation of increasing planetary data and novel concepts of biosignatures (Walker et al., 2018 in this issue); and (5) a review of the upcoming telescope capabilities to characterize exoplanets and their environment (Fujii et al., 2018 in this issue). Because of the immense content of these review papers, this summary provides a guide to their complementary scope and highlights salient features. Strong themes that emerged from the workshop were that biosignatures must be interpreted in the context of their environment, and that frameworks must be developed to link diverse forms of scientific understanding of that context to quantify the likelihood that a biosignature has been observed. Models are needed to explore the parameter space where measurements will be widespread but sparse in detail. Given the technological prospects for large ground-based telescopes and space-based observatories, the detection of atmospheric signatures of a few potentially habitable planets may come before 2030. Key Words: Exoplanets-Biosignatures-Remote observation-Spectral imagingBayesian analysis. Astrobiology 18, 619-629.
\end{abstract}

${ }^{1}$ NASA Goddard Institute for Space Studies (GISS), New York, New York, USA

${ }^{2}$ Nexus for Exoplanet System Science, ROCKE-3D Team, NASA GISS, USA.

${ }^{3}$ NASA Astrobiology Institute, Virtual Planetary Laboratory, University of Washington, Seattle, Washington, USA.

${ }_{5}^{4}$ NASA Goddard Space Flight Center, Greenbelt, Maryland, USA.

${ }^{5}$ NASA Ames Research Center, Exobiology Branch, Mountain View, California, USA.

${ }^{6}$ Department of Earth and Space Sciences/Astrobiology Program, University of Washington, Seattle, Washington, USA.

${ }^{7}$ Earth-Life Science Institute, Tokyo Institute of Technology, Ookayama, Meguro, Tokyo, Japan.

${ }^{8}$ Astronomy Department, University of Washington, Seattle, Washington, USA.

${ }^{9}$ Department of Earth Sciences, University of California, Riverside, California, USA.

${ }^{10}$ NASA Postdoctoral Program, Universities Space Research Association, Columbia, Maryland, USA.

${ }^{11}$ Blue Marble Space Institute of Science, Seattle, Washington, USA.

${ }^{12}$ School of Earth and Space Exploration, Arizona State University, Tempe, Arizona, USA.

${ }^{13}$ Beyond Center for Fundamental Concepts in Science, Arizona State University, Tempe, Arizona, USA.

${ }^{14}$ ASU-Santa Fe Institute Center for Biosocial Complex Systems, Arizona State University, Tempe, Arizona, USA.

(C) Nancy Y. Kiang et al., 2018; Published by Mary Ann Liebert, Inc. This Open Access article is distributed under the terms of the Creative Commons Attribution Noncommercial License (http://creativecommons.org/licenses/by-nc/4.0/) which permits any noncommercial use, distribution, and reproduction in any medium, provided the original author(s) and the source are credited. 


\section{Introduction}

Ince Voyager 1's image of Earth at less than pixel size in 1990 (NASA/JPL-CalTech, ${ }^{1}$ 1990; Sagan, 1994) and the Galileo spacecraft's flyby of Earth with detailed imagery and spectra (Sagan et al., 1993), the prospect of detecting life beyond Earth has inspired community interchange across scientific disciplines. There is a history of workshops designed to identify signs of life_- "biosignatures"- - and ways to find them. These have included the Pale Blue Dot workshops held in 1996, 1999, and 2006; workshops for the NASA Astrobiology Roadmaps (NASA, 1998; NASA, 2003; Des Marais et al., 2008) and NASA Astrobiology Strategy 2015 (Hays, 2015); and the European AstRoMap project 2013-2015 (Horneck et al., 2016), which was the first to lay out an astrobiology roadmap for the European Union. These workshops have defined program goals and research priorities, covering planet formation, habitability, prebiotic chemistry, coevolution of life and the host planet, in situ biosignatures on Solar System planetary bodies, and observational methods. However, there has not been a major comprehensive review devoted to the science and technology of remotely detectable biosignatures relevant to exoplanets since that of Des Marais et al. (2002).

The rapid rate of discoveries of exoplanets has expanded the scope of the science possible, as the exoplanets that have been discovered exhibit properties that suggest a diversity of worlds far beyond that seen in our solar system. In order to probe that diversity, exoplanet scientists plan to assess the chemical composition of exoplanets, along with information on planetary surface composition, to seek out signs of life on potentially habitable exoplanets using telescopes. Multiple observatories are currently being conceptualized with the explicit goal to search for exoplanet biosignatures. This has brought about a need for an integrative assessment of the state of exoplanet biosignature science.

In response to this need, the Exoplanet Biosignatures Workshop Without Walls (EBWWW) was organized in 2016. The EBWWW was designed as a series of community activities to engage the international astrobiology, exoplanet, and mission-concept communities to review, discuss, debate, and advance the science of remotely detectable exoplanet biosignatures. The activities were sponsored by the NASA Nexus for Exoplanet System Science (NExSS), ${ }^{2}$ a program formulated in 2015 in response to the rising need to enable support for exoplanet science across NASA's Astrophysics, Earth Science, Heliophysics, and Planetary Science divisions, as well as to enable international collaboration. The workshop also had a goal to produce a report for a Study Analysis Group (SAG) of the NASA Exoplanet Exploration Program Analysis Group (ExoPAG), which serves as the mode of community review for exoplanet research directions. Given the highly interdisciplinary nature of the science and technology for remote detection of life on exoplanets, a Scientific Organizing Committee (SOC) was formed to provide international and intellectually diverse representation. NExSS and the NASA Astrobiology Institute (NAI) provided vital communication tools and logistics for a

\footnotetext{
${ }^{1} \mathrm{NASA}=$ National Aeronautics and space Administration; JPL $=\mathrm{Jet}$ Propulsion Laboratory; CalTech = California Institute of Technology. ${ }^{2}$ NExSS website: http://www.nexss.info/
}

workshop format designed to foster interdisciplinary interchange between participants, to educate individual scientists on perspectives outside their field, and to nurture advanced graduate students and postdoctoral researchers in adopting broad interdisciplinary approaches.

Activities commenced with a series of interactive online videoconferences to engage participants across time zones around the globe. The meetings included introductory lectures and involved the participants in summarizing the state of biosignature science and reviewing observation technology. These virtual meetings served as prelude to a 3-day working meeting in Seattle, Washington, involving both inperson and unlimited remote participants to discuss advancing the science and developing conceptual frameworks and confidence standards for assessing the likelihood of life being manifested in the observation of a planet.

Five review papers resulted from the workshop and are presented in this issue of Astrobiology. These are the culmination of campaigns to solicit community input, including an online discussion forum on the NExSS website. Thus, these papers represent an international scientific communitywide review of the science and observational technology of exoplanet biosignatures to date. The papers provide guidance for life-detection missions currently being studied, and present a pathway for development of the field that can be pursued over the next few decades.

The five articles in this issue attempt a comprehensive treatment of the questions and concepts surrounding the search for life on exoplanets. They provide (1) an encyclopedic review of known and proposed biosignatures and models used to ascertain them (Schwieterman et al., 2018 in this issue); (2) a review of $\mathrm{O}_{2}$ as a biosignature, detailing its nuances as an indepth example of the contextual knowledge required rigorously to assess any claims of life on exoplanets (Meadows et al., 2018 in this issue); (3) a comprehensive approach to organize current qualitative understanding and available data into a formal quantitative Bayesian framework (Catling et al., 2018 in this issue); (4) an extension of that Bayesian framework in anticipation of increasing planetary data and the need to incorporate other conceptual frameworks for biosignature assessment both for traditional biosignatures and potential new avenues (Walker et al., 2018 in this issue); and (5) a review of the upcoming technological capabilities_-both planned and possible - that could provide the data needed to search for exoplanet biosignatures (Fujii et al., 2018 in this issue).

These articles chart a course toward detection of life within an encompassing quantitative framework. A strong community consensus that emerged from the NExSS EBWWW is that potential signs of life must be understood within the context of the environment, measurements of which should be provided by a variety of observation techniques. Quantification of the likelihood that a biosignature has been observed requires frameworks that link the potential biosignature to the environmental context. Figure 1 illustrates how the field is evolving from searching for specific products of life toward developing probabilistic frameworks that encompass whole systems. These developments will constitute much of the future research in the field, with regard to integrating diverse types of scientific understanding and interpreting statistics of sparse data. Ultimately, such frameworks will incorporate the latest research and have the ability to reassess biosignatures as new data and concepts emerge. This summary of the review 


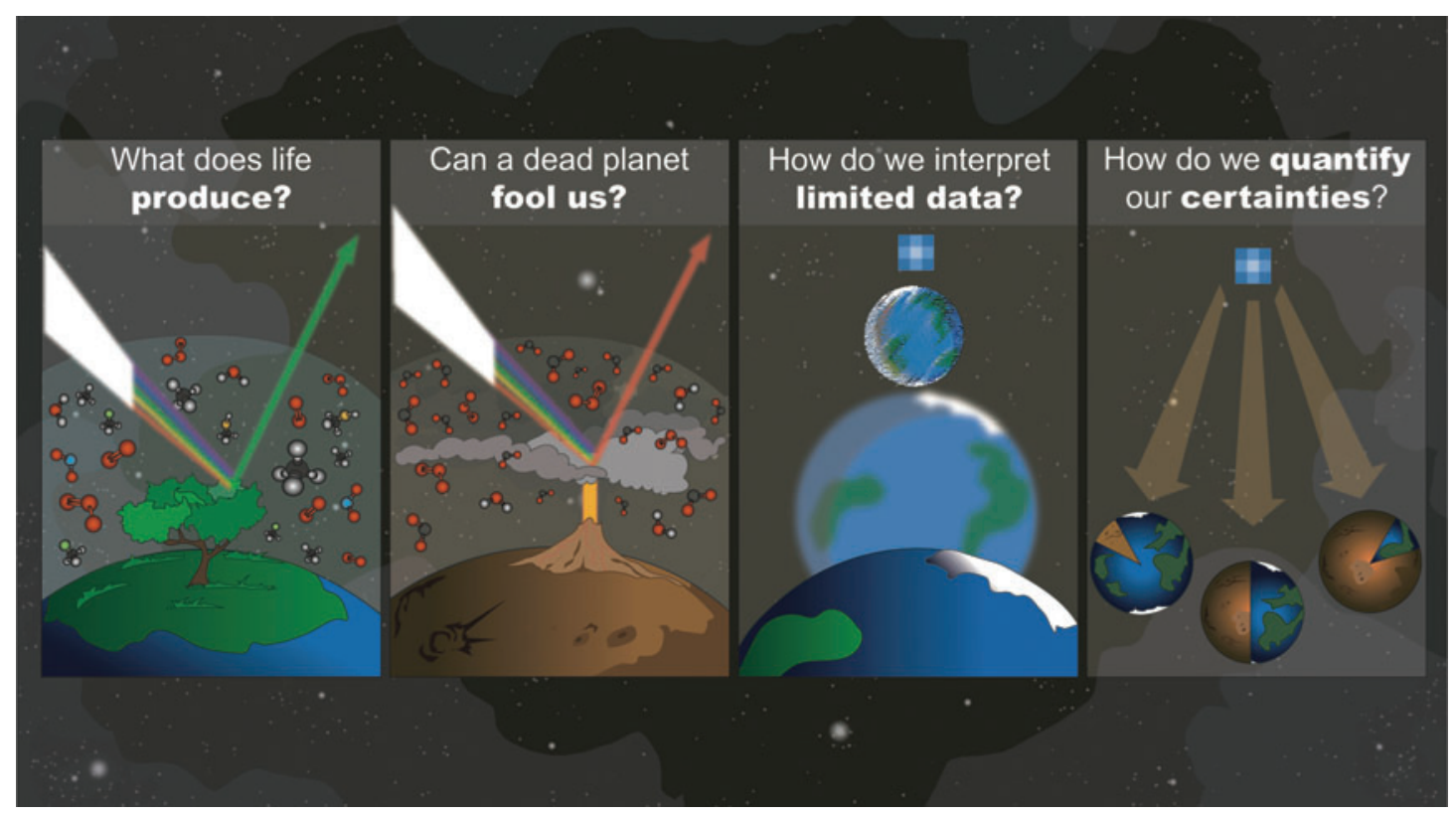

FIG. 1. An overview of the past, present, and future of research on remotely detectable biosignatures. Research historically has focused on cataloguing lists of substances or physical features that yield spectral signatures as indicators of potential life on exoplanets. Recent progress has led to an understanding of how environmental context is critical to interpret signatures of nonliving planets that may mimic some effects of biota. Exoplanet observing telescopes in the near future hold promise to provide direct spectral imaging that can chemically characterize rocky planets in the habitable zone of their parent star. Anticipating these capabilities, the field should seek to develop frameworks to utilize widespread but sparse data to deliver quantitative assessments of whether or not a given planet has life. (Credit: Aaron Gronstal)

series is intended to highlight key findings, serve as a guide for navigating their large content, and hopefully inspire those researchers interested in astrobiology-from students to senior scientists-to explore and collaborate outside the comfort of their individual disciplines.

\section{What Is a Biosignature?}

Life alters the chemistry of a planet. Spacecraft have observed the biological alteration of modern Earth's global spectrum in ways that should also be detectable across interstellar space but very coarsely resolved by telescopes. A "biosignature" may broadly be defined as "an object, substance, and/or pattern whose origin specifically requires a biological agent" (Des Marais and Walter, 1999; Des Marais et al., 2008), with further discussion in the work of Schwieterman et al. (2018 in this issue) of various ways the term may be used or understood. Catling et al. (2018 in this issue) generalize the definition to "any phenomenon, substance, or group of substances that provides evidence of the presence of life.' Such biosignatures include biogenic gases that affect the chemistry of Earth's atmosphere, as well as changes to surface properties of the planet due to organisms living in the ocean and on land. The previous major review of this topic by Des Marais et al. (2002) identified several accepted biosignature candidates as targets for observation as global-scale signs of life. These include biosignature gases in the atmosphere, oxygen $\left(\mathrm{O}_{2}\right)$, ozone $\left(\mathrm{O}_{3}\right)$, methane $\left(\mathrm{CH}_{4}\right)$, nitrous oxide $\left(\mathrm{N}_{2} \mathrm{O}\right)$, and methyl chloride $\left(\mathrm{CH}_{3} \mathrm{Cl}\right)$; and a surface biosignature, the vegetation "red edge," a unique reflectance spectrum from plant leaves and a sign of oxygenic photosynthesis. Since that early review by Des
Marais et al. (2002), researchers have probed the efficacy of these signs of biogenic gases and photosynthesis for exoplanets and identified additional candidates. Schwieterman et al. (2018 in this issue) present an exhaustive review of the mechanisms, sources, sinks, and environmental by-products for gas and surface biosignature candidates to date.

A major aspect of the review by Schwieterman et al. is the contribution of coupled photochemical/climate modeling studies that have been key in simulating the self-consistent environmental context for these gases. Such models have identified scenarios in which biogenic gases that are not persistent in Earth's atmosphere could be significant and detectable in other planetary and stellar contexts (Segura et al., 2005). These models can also be used to investigate scenarios of how alternative biosignatures might emerge as a result of environmental interactions. Of particular concern for astrobiologists has been the lack of a clear biosignature for a living planet that does not have oxygenic photosynthesis. An example of this is Earth during the Archean Eon ( 4.0-2.5 Gya), when anoxygenic photosynthetic bacteria and other anaerobic microbes were prevalent. Theoretical studies have identified potential atmospheric biosignatures for an anoxic planet, including an orange-hued organic haze (Arney et al., 2016) and ethane $\left(\mathrm{C}_{2} \mathrm{H}_{6}\right.$; (Domagal-Goldman et al., 2011). Both of these potential biosignatures would be photochemical by-products of the release of other gases, such as methane and sulfurbearing organic gases. (This is similar to how $\mathrm{O}_{3}$ is a potential sign of biological $\mathrm{O}_{2}$ production.) Schwieterman et al. additionally review studies that investigate false-positive cases for $\mathrm{O}_{2}$ as a biosignature, detectability of biosignatures in $\mathrm{H}_{2}$ dominated atmospheres, and the numerous modeling studies of the effects of $\mathrm{M}$ dwarf spectral radiation. 
Future modeling studies will have a new wealth of other biogenic gases to explore: a recent major scouring of the literature and chemical databases has identified $\sim 14,000$ small volatile molecules, of which a fourth are produced by life and the others potentially so (Seager et al., 2016). This dramatically expands the library of candidate biosignature gases and should motivate a large-scale effort at further indepth characterization, both in the laboratory and in understanding the sources and fates of these gases. Meanwhile, recent work has revisited the old Lovelock (1965) proposal that atmospheric chemical disequilibrium indicates life. Rigorous quantification of chemical disequilibrium of the atmospheres of Solar System planets demonstrates an elegant approach to discerning a biosphere versus a lifeless planet from remotely measurable data (Krissansen-Totton et al., 2016, 2018).

For surface biosignatures, with regard to photosynthesis, Schwieterman et al. provide a review of the general principles of light harvesting and charge separation by which photosynthetic organisms convert light to chemical energy. Such signatures may not be inherently tied to a specific wavelength; instead, inferring the presence of photosynthesis requires a process-based understanding of how light-harvesting pigments may tune their absorption to the light emitted by different stellar types from the Sun. A catalogue and classification of pigments is provided, for both oxygenic and anoxygenic photosynthesis. Among these pigments are novel chlorophylls that have recently been discovered (Miyashita et al., 1997; Chen et al., 2010), which now expand the parameter space for adaptation of oxygenic photosynthesis to alternative spectral environments. The authors review modeling studies that have attempted to quantify the detectability of surface photosynthetic signatures and possible alternative adaptations or evolutionary stages. Such studies have investigated the vegetation red edge in the Earthshine and in disk-integrated spectra, a near-IR "edge" that might dominate on an M star planet, and even an Archean "purple Earth" due to purple anoxygenic phototrophs (Montanes Rodriguez et al., 2004; Tinetti et al., 2006; Kiang et al., 2007b; Arnold et al., 2009; Robinson et al., 2011; Sanromá et al., 2014; Takizawa et al., 2017).

Schwieterman et al. also summarize numerous potential surface biosignatures involving pigments with functions other than photosynthesis, such as photo-protection and ecological signaling (DasSarma, 2006; Hegde et al., 2015; Schwieterman et al., 2015a). Future work will require further vetting of all pigment signatures against a mineral or rock background to improve confidence in their interpretation.

Other kinds of biosignatures were proposed or discussed at the workshop but have not yet been studied in as much detail as gaseous and pigment biosignatures. The temporal and even geographic variation of biosignature signal strengths and surface exchanges with the atmosphere will increasingly be probed with tools like three-dimensional spectral simulators and general circulation models (GCMs). The chirality of biomolecules is a strong indicator of life (Sparks et al., 2012); however, detection of weak signals from chiral biosignatures requires further development of the science and technology of spectropolarimetry.

\section{Oxygen as Our Most Robust Biosignature: Ruling out False Positives and Negatives}

Oxygen remains our most robust biosignature and the most studied. Meadows et al. (2018 in this issue) provide an end-to-end review of oxygen as a biosignature, from its origins to its detection, covering unknowns about the evolution of oxygenic photosynthesis on Earth; the rise of atmospheric oxygen; the abiotic processes that also can produce oxygen; the variety of environmental contexts that can produce false negatives and false positives, as well as definitive answers; and an exhaustive survey of measurement techniques and critical wavelengths to provide both direct and contextual information.

While the abundant $\mathrm{O}_{2}$ (and its by-product $\mathrm{O}_{3}$ ) in Earth's modern atmosphere is the most easily detectable feature of life on Earth, the history of oxygen on our planet includes periods when life was present but oxygen existed at undetectable levels. It may even have been possible that oxygenic photosynthesis persisted for billions of years before oxygen accumulated to detectable levels in the atmosphere (Lyons et al., 2014; Reinhard et al., 2017). Current unsettled questions that challenge our ability to determine the likelihood for oxygenic photosynthesis to generate a detectable signal on an exoplanet include: the likelihood of the innovation of oxygenic photosynthesis in another planetary setting given the environmental selection pressures; the origin of the $\mathrm{Mn}_{4} \mathrm{CaO}_{5}$ cluster, the unique biomolecule that performs water oxidation and generates $\mathrm{O}_{2}$ (Blankenship and Hartman, 1998; Cardona, 2016; Fischer et al., 2016); and the biogeochemical complexities involved in the accumulation of atmospheric $\mathrm{O}_{2}$ (Lyons et al., 2014).

Current understanding strongly suggests that atmospheric $\mathrm{O}_{2}$ is not always an unambiguous biosignature on a habitable planet, as recent work has shown myriad ways that false positives can be generated. Critically, all of them depend on eliminating the reducing gases that would otherwise consume oxygen $(\mathrm{Hu}$ et al., 2012; Tian et al., 2014; Wordsworth and Pierrehumbert, 2014; Gao et al., 2015; Harman et al., 2015; Luger and Barnes, 2015; Narita et al., 2015). There are several known ways to discriminate between biological and nonbiological production of $\mathrm{O}_{2}$ by using the environmental context. One is to search for the presence of reduced gases, such as methane $\left(\mathrm{CH}_{4}\right)$. The presence of $\mathrm{CH}_{4}$ could indicate rapid destruction of oxygen in that atmosphere and a requirement for fast replenishment of the $\mathrm{O}_{2}$ that is most likely met by biological activity. Another method is to analyze the stellar environment: high UV radiation from the host star, particularly active $M$ dwarf stars, can increase abiotic $\mathrm{O}_{2}$ and $\mathrm{O}_{3}$ production from either atmospheric escape of $\mathrm{H}$ or rapid photolysis of $\mathrm{CO}_{2}$. For $\mathrm{F}$, $\mathrm{G}$, and $\mathrm{K}$ stars that emit less $\mathrm{UV}$, abiotic $\mathrm{O}_{2}$ and $\mathrm{O}_{3}$ is limited to particular contexts, such as those lacking water vapor, or lacking background $\mathrm{N}_{2}$ and cloud formation (Wordsworth and Pierrehumbert, 2014).

Meadows et al. (2018 in this issue) note that the sinks for $\mathrm{O}_{2}$ and $\mathrm{O}_{3}$, such as weathering, and aqueous sinks for $\mathrm{CO}$, are as yet poorly understood, so the quantitative results of these modeling studies need further constraints and further coupling of atmospheric models with ocean chemistry and surface weathering models. The authors emphasize yet more known nuances of false-positive cases as well as false negatives. They then scrutinize how to observe for them with regard to detectability of particular gases (Schwieterman et al., 2015b, 2016) as well as surface biosignatures arising from biological pigments in the absence of clear 
atmospheric signals, such as those summarized in the previous section from Schwieterman et al. (2018 in this issue).

This work on oxygen carries a strong lesson that astrobiologists must also apply to other proposed biosignatures: they must be interpreted with respect to their environmental context. Oxygen's status as a relatively unassailable biosignature has been tempered now by cases of false positives, but in well-defined scenarios that can lend greater confidence to the true positives. The astrobiology and exoplanet modeling communities should apply the same rigor of assessment to other proposed biosignatures and consider the full range of planetary contextual measurements that would be required to confirm their biogenicity. The data on the environmental context will not necessarily be complete and will itself be subject to uncertainties, which brings us to the next paper in this series.

\section{Frameworks to Quantify the Probabilities for Life}

To date, much of the theory of biosignatures is described in qualitative terms, and the associated advice to mission/ instrument design teams is similarly qualitative. For example, it is acknowledged that the confirmation of biosignatures requires a comprehensive classification of the planetary environment, which in turn leads to a suggestion to obtain observations with as broad a wavelength range as possible. But detailed trade-offs for specific instruments, and eventually the interpretation of data from biosignature searches, will be best enabled by a more quantitative probabilistic framework.

A major challenge in such quantification is that assessing the presence or absence of life on a planet is an inherently complex problem, requiring comprehensive analyses of the planetary context, involving multiple systems that interact with each other, often in nonlinear ways. Accounting for this in a quantitative manner-and doing so in a way that is flexible enough to handle alien worlds with potentially alien climates and potentially alien life-requires an encompassing framework. Bayes' theorem, in particular, was identified at the EBWWW as having the potential to advance our field's ability to synthesize sparse data, and as a framework for combining understanding from diverse scientific disciplines.

According to Bayes' theorem, one can calculate the conditional probability that something is true, such as the likelihood of a system having a given property based on available data. Catling et al. (2018 in this issue) and Walker et al. (2018 in this issue) present complementary applications of the Bayesian framework to quantifying the likelihood that signs of life are present in an exoplanet data set.

The derivation by Catling et al. (2018 in this issue) is shown in Fig. 2 (adapted from Walker et al., 2018 in this issue). This particular derivation specifically dissects what might be observed ( $D=$ data) given either the presence or absence of life within a particular exoplanet environment context $(C=$ context $)$, that is, $P($ data $\mid$ context and life $)$ and $P($ data $\mid$ context and no life $)$, respectively. $P($ life $\mid$ context $)$ is a "prior" quantitative expression of likelihood of life given the context of the exoplanet. That "context" includes habitability afforded by the stellar and planetary system environment as well as scientific understanding derived from planetary and biosphere system models, and from Earth's history and diversity of life. Catling et al. (2018 in this issue) describe their framework using probabilities conditional on the planetary context because this method acknowledges that life and environment are interdependent. Obviously, life requires a suitable environment, such as a planet that supports liquid water. But the Bayesian logic also runs the other way: the presence of life constrains a planetary environment. For example, a planet with life could not be orbiting at $0.1 \mathrm{AU}$ distance around a Sun-like star.

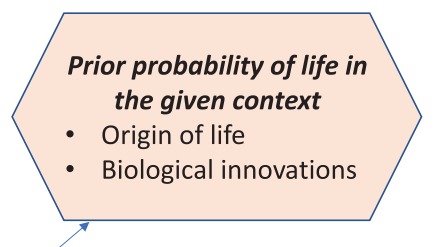

$$
P(\text { life } \mid D, C)=\frac{P(D \mid C, \text { life }) P(\text { life } \mid C)}{P(D \mid C, \text { no life })(1-P(\text { life } \mid C))+P(D \mid C, \text { life }) P(\text { life } \mid C)}
$$

\begin{tabular}{|l|}
\hline \multicolumn{1}{c|}{ Posterior } \\
detection of life \\
- Statistics \\
- Distribution of \\
biogeochemical states \\
- Frequency of biological \\
innovation \\
- Technology detection \\
biases
\end{tabular}

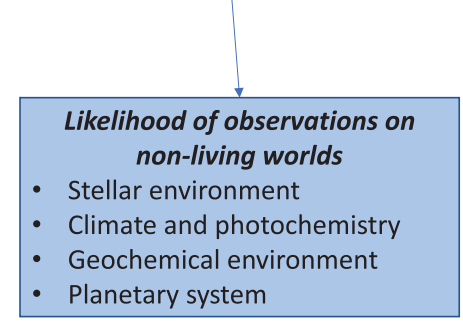

$\mathrm{D}=$ data/observations with potential biosignature(s)

$\mathrm{C}=$ exoplanet context

Probability logic symbols: P="probability of", |="given", comma="and"
Likelihood of observations on living worlds for concepts of life

- Black box approaches to living processes

- Improbable chemistry

- Co-evolution with the planet

- Universal biology: scaling laws, informational biosignatures, network biosignatures

FIG. 2. A Bayesian framework, applied to the detection of life on extrasolar planets. Equation from Catling et al. (2018 in this issue). Adapted from Walker et al. (2018 in this issue). 
Catling et al. then present an organization for currently accepted knowledge and understanding to make use of the above Bayesian framework. They offer a systematic scheme to assimilate how different variables of the stellar and planetary system environment and the planet's internal properties affect habitability, the development of life, and expression of biosignatures, and, especially valuable, how these properties may be measured remotely. The paper provides a concise catalogue of spectral features of gas and surface biosignature candidates, and presents a first review to date of potential false-positive cases for a wide variety of environmental factors. The authors also present a review of "anti-biosignatures," exemplified by gases, like $\mathrm{CO}$, or combinations of gases, such as $\mathrm{H}_{2}$ and $\mathrm{CO}_{2}$, that arise in contexts that would lack life (Zahnle et al., 2008; Gao et al., 2015). Finally, Catling et al. propose a framework for classifying confidence levels for the likelihood that a biosignature has been observed. This framework follows the example of the Intergovernmental Panel on Climate Change (IPCC) in their approach to communicate uncertainty about global warming of the Earth system (IPCC, 2014). Thus, the Bayesian framework derived by Catling et al. provides a clear path to organize the data and scientific understanding according to how models may synthesize the context for life and distinguish the different sources of uncertainty. The final posterior probability $P($ life $\mid$ data, context $)$ can be classified into the proposed confidence tiers for public communication.

\section{Expanding the Framework for the Search for Life}

While Catling et al. (2018 in this issue) provide a framework for synthesizing current knowledge and understanding, with the likelihood of life conditional upon the planetary context, $P$ (life $\mid$ data, context), Walker et al. (2018 in this issue) anticipate the impending accumulation of statistics of exoplanets and take the next step in the Bayesian framework to address: $P($ life $)$, the prior probability of life occurring at all elsewhere in the Universe. For lack of other examples than Earth, astrobiologists sometimes resort to drawing conclusions based on how quickly life emerged on Earth, but $P$ (life) is truly quantifiable only with large statistics, after more examples of life have already been discovered. Walker et al. confront the issues around quantification of $P($ life) itself and discuss how the search for life is confounded by lack of clarity on the very definition of life. They present a rough illustration of the vastly interdisciplinary nature of the search for life in a table of how different scientific disciplines characterize the nature and activity of life, from physical, chemical, systems, and many other perspectives. The challenges are illustrated in a discussion of the "Type" classification of biosignatures that was initiated by Seager et al. (2013). Considering the drive for a processbased approach, the authors discuss how $P($ life $)$ itself is decomposable into a family of probabilities for the emergence and existence of important, path-dependent life processes, such as particular metabolisms, multicellularity, and so on.

Given the continually evolving concepts of life, Walker et al. approach Bayesian analysis as a means to incorporate additional types and novel concepts of life, and to organize new data and understanding as they become available (Kim et al., 2018). For the detection of biology that differs from that of Earth's, the Bayesian framework offers a means to integrate the languages of a diverse community, each having its own frontiers of knowledge and understanding. For this, Walker et al. identify research areas with promise for probing fundamental unknowns to advance empirical and theoretical understanding for synthesis in complex models. These areas include acquisition of data on and modeling the role of stellar environments, particularly the UV, in atmospheric processes and observables; development of coupled geophysical and climate models, both 1D models and 3D GCMs; understanding how heat and gases from volcanoes and ocean vent systems regulate the planet's chemistry; accounting for biological processes that can lead to different biochemical adaptations other than those found on Earth; and concepts of "universal biology," wherein the emergent properties of a system may be quantified with respect to network theory, information theory, or statistics from ensemble GCM investigations. Network theory describes the structure of biological systems across micro- to macroscales, wherein the biological networks exhibit distinctive topology that could be a biosignature. Chemical distributions are altered by life in a variety of different ways. Catling et al. (2018 in this issue) review thermodynamic quantification, such as a chemical disequilibrium departing from an abiotic system (Krissansen-Totton et al., 2016), or a selective distribution in molecular weights of chemical species (Krissansen-Totton et al., 2018). Walker et al. (2018 in this issue) provide an information theoretic perspective on how chemical distributions could indicate the presence of natural selection, in which low entropy (McKay, 2011) or high abundance of complex molecules (Marshall et al., 2017) could be indicative of life. The authors further generalize the definition of "anti-biosignatures" as any phenomena that diminish the likelihood that a signal is generated by life.

In a helpful example exercise, Walker et al. draw upon Meadows et al. (2018 in this issue) to step through quantifying $\mathrm{O}_{2}$ as a biosignature. They demonstrate estimating the likelihood that oxygenic photosynthetic life has been observed in a data set, and how the probabilities change as new observations and understanding are incorporated. These explorations by Walker et al. advocate that the community move forward from identifying biosignatures as products or objects and instead consider biosignatures as expressions of underlying processes (including utilization of energy as well as evolutionary processes).

Both Catling et al. and Walker et al. emphasize the necessity of characterizing planetary systems both with and without the influence of life in order to quantify the likelihood, $P$ (life $\mid D, C$ ). The other worlds in our own solar system will serve as important resources to constrain the conditional probabilities. We have as guides detailed observations of Mars, Venus, and other planetary bodies, none of which as yet are known to express global biospheres, possibly, though not yet conclusively, providing us examples of $P$ (data $\mid$ no life). Guidance may also come from Earth history, as innovations in life processes impacted the planet on a global scale and changed the "appearance" of Earth through different eons, providing us examples for $P($ data $\mid$ life $)$.

This directly translates to what can be tackled with planetary models, of which different kinds are reviewed in the works of Schwieterman et al., Catling et al., and Walker 
et al. (2018 in this issue). These models can be used to simulate the given context both with and without the proposed life process, and thus explore how likely it would be for the biosignature candidate to be expressed, biotically or abiotically, as seen in the observed data. The limited sample of worlds in our solar system offers snapshots and templates for evaluating the level of our understanding in process-based physical models. After such model validation, researchers can then introduce perturbations: alternative stellar radiation, planetary parameters, and life processes. These capabilities will enable scientists to theorize about potential features of exoplanets and ultimately to explain those that will be observed.

The completeness of the Bayesian equation implies that the astrobiology community needs comprehensive and diverse models of living planets and similar models of nonliving planets, as well as advancement of our knowledge and understanding thus far of the likely emergence of life or particular life processes and their impact on the environment. The Bayesian approach affords the synthesis of diverse areas of knowledge into a framework that quantifies probabilities, rather than imposing only one system of knowledge; for example, the predicted values from the physics of a GCM ensemble of experiments can be combined with the likelihood of other phenomena inferred from the fossil record. It is particularly useful for identifying the terms most challenging to quantify. It is also a means to account for uncertainty in measurements (Parviainen, 2017) and thus tune search strategies. For example, it permits quantitatively assessing whether targeted searches or broader surveys will provide more confidence in detection of life (Walker et al., 2018 in this issue). Given the highly interdisciplinary nature of the search for exoplanet biosignatures, adoption of a Bayesian concept is encouraged to help scientists work across disciplines, identify the significance of critical unknowns, and provide quantitative assessments of confidence in scientific conclusions. The Bayesian framework offers a means to completeness in quantifying the probabilities in a variety of contexts and modes of understanding, and moreover allows that there is, of course, no unique way to express the problem, which will evolve as more data change our understanding.

\section{Observational Technology: Capabilities in the Near Future and Beyond}

As the research outlined above progresses, so will understanding of planets from ongoing exoplanet observations, which will continue to improve as new observational capabilities come online. A recent review by Guyon (2017) provides a concise catalogue of observational techniques to detect exoplanets: transit photometry, microlensing, radial velocity, astrometry, interferometry, and coronagraphy. Fujii et al. (2018 in this issue) extend this further, giving weight to the techniques to characterize the internal properties of potentially habitable planets, that is, not only their sizes and orbits but also their atmospheres and surfaces, which are necessary for detection and evaluation of biosignature candidates.

Fujii et al. (2018 in this issue) present an extensive review of the sweep of exoplanet observation missions-past, current, and in the near future-and the evolution of their target goals for properties of rocky planets, in three eras. The first era has been focused on the astrophysical characterization of planetary orbits and sizes (e.g., using groundbased surveys, CoRoT, Kepler, K2, Gaia, CHEOPS, TESS, and later PLATO, WFIRST) ${ }^{3}$. The second era will focus on the chemical characterization of atmospheres of a wide variety of exoplanets, most of which are uninhabitable gaseous planets (e.g., Hubble, Spitzer, James Webb Space Telescope [JWST], the ground-based Extremely Large Telescopes [ELTs], and missions in development). The third era will focus on the astrobiological characterization of the life and habitable environments that exist on exoplanets (possibly JWST, and missions in development). Fujii et al. (2018 in this issue) provide a table of planned observatories, both space-based and ground-based, comparing their expected start and technical features and capabilities.

The article by Fujii et al. serves as an in-depth reference on the abilities of both space-based and ground-based telescopes to measure the broad range of parameters that are relevant to an assessment of the likelihood of life. These include the environmental context variables that affect habitability, chemistry, and the very interpretation of biogenicity, e.g., physical properties of planets (mass, radius, orbit), atmospheric inventory, surface liquid water, other surface materials, thermal profiles, planetary spin, heterogeneity, stellar radiation, and the role of neighboring planets for insights into planet formation.

The most significant trend in development of exoplanet observational capability is the real impending prospect to measure spectroscopic properties of planets with sizes within the range for rocky composition and in the habitable zone with their parent stars. Transit photometry missions such as Kepler/K2, and radial velocity techniques, have ballooned the population statistics, showing that Earth-sized planets are not rare, with improved statistics, particularly for late-type, cool stars. Ground observations have also identified and will continue to identify a few "golden targets"rocky planets in the stellar habitable zone that are close enough to Earth for spectroscopic follow-up. Such discoveries will accelerate with continued observations from the ground and with the launch of the Transiting Exoplanet Survey Satellite (TESS) in 2018. Both of these missions will find more nearby targets for follow-up transit spectroscopy analyses.

Attempts to further investigate these "golden targets" might begin with JWST (2020-) and will accelerate with next-generation ground-based telescopes possessing highresolution and/or high-contrast instruments (GMT, TMT, ELT: 2020s- $)^{4}$. These ELTs are planned to search for biosignatures on planets around M-type stars, with the caveat that ground-based detection of any such biosignatures must account and correct for the intervening gases, including biosignature gases, in Earth's atmosphere. Confirmation of any ground-based detections of biosignatures could occur with next-generation space-based flagship telescopes, such as the

\footnotetext{
${ }^{3}$ CoRoT $=$ Convection, Rotation and planetary Transits; CHEOPS $=$ CHaracterising ExOPlanet Satellite; TESS = Transiting Exoplanet Survey Satellite; PLATO = PLAnetary Transits and Oscillations of stars; WFIRST $=$ Wide Field InfraRed Survey Telescope.

${ }^{4}$ GMT = Giant Magellan Telescope; TMT = Thirty-Meter Telescope; ELT = European-Extremely Large Telescope.
} 
Origins Space Telescope (OST), the Habitable Exoplanet Observatory (HabEx), the Large UV/Optical/IR Surveyor (LUVOIR). All of these mission concepts would be capable of conducting transit spectroscopy measurements from space, with a wavelength range that complements that planned for ground-based telescopes.

In addition to transit spectroscopy, HabEx and LUVOIR would also be able to conduct direct imaging of rocky planets, with capabilities to be advanced by technologies being developed for the Wide Field Infra-Red Space Telescope (WFIRST). Direct imaging is a powerful technique that would allow for some inventory of the atmosphere, including biosignatures for both oxygenated and anoxic worlds, contextual information to account for false positives, and surface characteristics. For the latter, variability with the planet's rotation may even allow characterization more detailed than a disk average, discerning the distributions of different surface types, such as continents and oceans (e.g., Fujii and Kawahara, 2012; Cowan and Strait, 2013). Space-based direct imaging would also allow the biosignature search to extend to planets in the habitable zones of Sun-like stars. This is important because transit spectroscopy measurements will be limited to planets with short orbital periods in the habitable zones of stars much cooler than the Sun, for which there are habitability concerns due to the high-energy environment created by these active stars (e.g., Airapetian et al., 2016, Garcia-Sage et al., 2017). The potential for such false positives is lessened around Sun-like stars where habitable zone planets will be less subject to intense stellar activity.

Fujii et al. (2018 in this issue) evaluate the efficacy of each of these techniques and missions with respect to their biases by stellar type, and by photometric and spectral sensitivities and discuss the extent to which they can discern gases of interest, the vertical structure of a planet's atmosphere, and surface conditions. The authors emphasize the importance of combining the capabilities of different techniques and missions to obtain data on the environmental context for biosignature observations to ensure the ability to rule out false positives. While some key parameters needed for model analyses will be a challenge to obtain, the authors offer a timeline for the technological developments and which data will become available at what time. Future missions in development would observe planets in the habitable zone of brighter, solar-type stars and aim to discover an Earth twin in orbit around a G-type star like the Sun. The overall conclusion of Fujii et al. is that "it is probably not unreasonable to expect the detection of the atmospheric signatures of a few potentially habitable planets to come before 2030," ushering in a "golden era of comparative planetology of terrestrial planets" and encouraging the development of frameworks to interpret the planetary environment from what can be observed.

\section{Conclusions}

These five major review papers were a community endeavor and aimed to rigorously and thoroughly summarize progress to date in the remote search for life on exoplanets. The papers further identify intellectual developments necessary to design new observational strategies and interpret the influx of new data from current and upcoming obser- vatories. The importance of environmental context in inferring biosignatures, the need to assess confidence in biosignature observations, and quantitative frameworks to integrate understanding across diverse scientific disciplines are all key areas for development in the field.

Further interactions along these lines will promote progress. In particular, the field is currently dominated by astronomers and would benefit from more input from origins-of-life researchers, planetary scientists, biologists, and early Earth biogeochemists to continue developing process-based approaches to characterizing exoplanet biosignatures and, moreover, their likelihoods. It is not sufficient simply to identify a molecule or a spectral feature for a biosignature. Rather, both gaseous and surface biosignature candidates require biological and biogeochemical understanding to anticipate the variety of ways these features may be expressed in different environmental contexts. Additionally, the communities that study exoplanets and those that study in situ biosignatures will benefit from greater communication to share "lessons learned" and seek common approaches to the unifying problem of quantitative assessment of biosignature detection. Statistical approaches are strongly advocated for quantifying confidence levels and the development of confidence standards for exoplanet biosignature evaluation, such as those proposed in these review papers. A Bayesian probability framework, in particular, allows for quantitative synthesis of the qualitative understanding of diverse scientific disciplines, to help with instrument/ mission design strategy, observation planning, and eventually analysis of data that will be wide in sampling but sparse in detail.

The search for life beyond our solar system has a breathtaking momentum, and these review papers propose frameworks to advance the field in multiple ways that take into account the complex interactions of a biosphere with its host planet and star. The estimated probabilities of life given the observations will be invaluable for communicating the results of our efforts. To scientific peers searching for life on planets around other stars, these concepts will provide comparative tools for evaluating different proposed biosignatures for observed targets. For public communication, these frameworks provide a rigorous test of confidence before announcing that we are not alone.

\section{Acknowledgments}

The Exoplanet Biosignatures Workshop Without Walls (EBWWW) could not have been possible without the support of Mary Voytek, director of NASA's Astrobiology Program and the Nexus of Exoplanet System Science, and Carl Pilcher and Penny Boston, former and current directors of the NASA Astrobiology Institute. Thanks are also due to the many scientists who led the several cross-disciplinary discussion activities of the workshop. The Science Organizing Committee: Daniel Apai, Shawn Domagal-Goldman (co-chair), Yuka Fujii, Lee Grenfell, Nancy Y. Kiang (cochair), Adrian Lenardic, Nikole Lewis, Timothy Lyons, Hilairy Hartnett, Bill Moore, Enric Pallé, Niki Parenteau (co-chair), Heike Rauer, Karl Stapelfeldt, Sara Walker. Online discussion and in-person workshop speakers and participants: SOC named above as well as Giada Arney, William Bains, Robert Blankenship, David Catling, Charles 
Cockell, David Crisp, Sebastian Danielache, Shiladitya DasSarma, Russell Deitrick, Anthony Del Genio, Drake Deming, Steve Desch, David Des Marais, Theresa Fisher, Sonny Harman, Erika Harnett, Siddharth Hegde, Yasunori Hori, Renyu Hu, Betül Kaçar, Jeremy Leconte, Andrew Lincowski, Rodrigo Luger, Victoria Meadows, Adam Monroe, Norio Narita, Christopher Reinhard, Sarah Rugheimer, Andrew Rushby, Edward Schwieterman, Nick Siegler, Evgenya Skolnick, Harrison Smith, Motohide Tamura, and Margaret Turnbull.

We also wish to thank the numerous other scientists who contributed valuable input and energy, remotely participating in discussions and providing comments in the EBWWW online discussion forum. We are grateful to Mike Toillion and Julia Figliotti for nimble responses in ensuring our online tools smoothly enabled interactions among geographically widely distributed community members.

The work on this paper was supported by: the NASA Astrobiology Program's Nexus for Exoplanet System Science (NExSS) funding for the ROCKE-3D (Resolving Orbital and Climate Keys of Earth and Extraterrestrial Environments with Dynamics) team (node 13-13NAI7_2-0007) and the Planet Formation team at Arizona State University; the NASA Astrobiology Institute's (NAI) teams, Virtual Planetary Laboratory (Cooperative Agreement Number: CAN NNA13AA93A) and Alternative Earths (CAN NNA15BB03A); NASA's internal science funding of the Sellers Exoplanet Environments Collaboration (SEEC); and the NASA Postdoctoral Program, Universities Space Research Association. Financial support for Open Access page charges was provided by NExSS.

\section{References}

Airapetian, V.S., Glocer, A., Gronoff, G., Hebrard, E., and Danchi, W. (2016) Prebiotic chemistry and atmospheric warming of early Earth by an active young Sun. Nat Geosci 9:452-455.

Arney, G., Domagal-Goldman, S.D., Meadows, V.S., Wolf, E.T., Schwieterman, E., Charnay, B., Claire, M., Hébrard, E., and Trainer, M.G. (2016) The pale orange dot: the spectrum and habitability of hazy Archean Earth. Astrobiology 16:873-899.

Arnold, L., Bréon, F.-M., and Brewer, S. (2009) The Earth as an extrasolar planet: the vegetation spectral signature today and during the last Quaternary climatic extrema. International Journal of Astronomy 8:81-94.

Blankenship, R.E. and Hartman H. (1998) The origin and evolution of oxygenic photosynthesis. Transactions in Biological Science (TIBS) 23:94-97.

Cardona, T. (2016) Reconstructing the origin of oxygenic photosynthesis: do assembly and photoactivation recapitulate evolution? Front Plant Sci 7, doi:10.3389/fpls.2016.00257.

Catling, D.C., Krissansen-Totton, J., Kiang, N.Y., Crisp, D., Robinson, T.D., DasSarma, S., Rushby, A.J., Del Genio, A., Bains, W., and Domagal-Goldman, S. (2018) Exoplanet biosignatures: a framework for their assessment. Astrobiology 18:709-738.

Chen, M., Schliep, M., Willows, R.D., Cai, Z.L., Neilan, B.A., and Scheer, H. (2010) A red-shifted chlorophyll. Science 329: 1318-1319.

Cowan, N.B. and Strait, T.E. (2013) Determining reflectance spectra of surfaces and clouds on exoplanets. Astrophys $J$ 765:L17.

DasSarma, S. (2006) Extreme halophiles are models for astrobiology. Microbe 1:120-126.
Des Marais, D.J. and Walter, M.R. (1999) Astrobiology: exploring the origins, evolution, and distribution of life in the Universe. Annu Rev Ecol Syst 30:397-420.

Des Marais, D.J., Harwit, M.O., Jucks, K.W., Kasting, J.F., Lin, D.N., Lunine, J.I., Schneider, J., Seager, S., Traub, W.A., and Woolf, N.J. (2002) Remote sensing of planetary properties and biosignatures on extrasolar terrestrial planets. Astrobiology 2:153-181.

Des Marais, D.J., Nuth, J.A., III, Allamandola, L.J., Boss, A.P., Farmer, J.D., Hoehler, T.M., Jakosky, B.M., Meadows, V.S., Pohorille, A., Runnegar, B., and Spormann, A.M. (2008) The NASA Astrobiology Roadmap. Astrobiology 8:715-730.

Domagal-Goldman, S.D., Meadows, V.S., Claire, M.W., and Kasting, J.F. (2011) Using biogenic sulfur gases as remotely detectable biosignatures on anoxic planets. Astrobiology 11: 419-441.

Fischer, W.W., Hemp, J., and Johnson, J.E. (2016) Evolution of oxygenic photosynthesis. Annu Rev Earth Planet Sci 44: 647-683.

Fujii, Y. and Kawahara, H. (2012) Mapping Earth analogs from photometric variability: spin-orbit tomography for planets in inclined orbits. Astrophys $J$ 755, doi:10.1088/0004-637X/ 755/2/101.

Fujii, Y., Angerhausen, D., Deitrick, R., Domagal-Goldman, S., Grenfell, J.L., Hori, Y., Kane, S.R., Palle, E., Rauer, H., Siegler, N., Stapelfeldt, K., and Stevenson, K.B. (2018) Exoplanet biosignatures: observational prospects. Astrobiology 18:739-778.

Gao, P., Hu, R., Robinson, T.D., Li, C., and Yung, Y.L. (2015) Stabilization of $\mathrm{CO}_{2}$ atmospheres on exoplanets around $\mathrm{M}$ dwarf stars. Astrophys J 806:249-261.

Garcia-Sage, K., Glocer, A., Drake, J.J., Gronoff, G., and Cohen, O. (2017) On the magnetic protection of the atmosphere of Proxima Centauri b. Astrophys J Lett 844. http://iopscience .iop.org/article/10.3847/2041-8213/aa7eca/meta.

Guyon, O. (2017) Habitable exoplanets detection: overview of challenges and current state-of-the-art. Opt Express 25: 28825-28837.

Harman, C.E., Schwieterman, E.W., Schottelkotte, J.C., and Kasting, J.F. (2015) Abiotic $\mathrm{O}_{2}$ levels on planets around F, G, $\mathrm{K}$, and $\mathrm{M}$ stars: possible false positives for life? Astrophys $J$ 812, doi:10.1088/0004-637X/812/2/137.

Hays, L., editor-in-chief. (2015) NASA Astrobiology Strategy 2015, NASA Headquarters, Washington, DC.

Hegde, S., Paulino-Lima, I.G., Kent, R., Kaltenegger, L., and Rothschild, L. (2015) Surface biosignatures of exo-Earths: remote detection of extraterrestrial life. Proc Natl Acad Sci USA 112:3886-3891.

Horneck, G., Walter, N., Westall, F., Grenfell, J.L., Martin, W.F., Gomez, F., Leuko, S., Lee, N., Onofri, S., Tsiganis, K., Saladino, R., Pilat-Lohinger, E., Palomba, E., Harrison, J., Rull, F., Muller, C., Strazzulla, G., Brucato, J.R., Rettberg, P., and Capria, M.T. (2016) AstRoMap European Astrobiology Roadmap. Astrobiology 16:201-243.

Hu, R., Seager, S., and Bains, W. (2012) Photochemistry in terrestrial exoplanet atmospheres. I. Photochemistry model and benchmark cases. Astrophys J 761, doi:10.1088/0004637X/761/2/166.

IPCC. (2014) Climate Change 2014 Synthesis Report: Summary for Policymakers, Intergovernmental Panel on Climate Change, Geneva, Switzerland.

Kiang, N.Y., Siefert, J., Govindjee, and Blankenship, R.E. (2007a) Spectral signatures of photosynthesis. I. Review of Earth organisms. Astrobiology 7:222-251. 
Kiang, N.Y., Segura, A., Tinetti, G., Govindjee, Blankenship, R.E., Cohen, M., Siefert, J., Crisp, D., and Meadows, V.S. (2007b) Spectral signatures of photosynthesis. II. Coevolution with other stars and the atmosphere on extrasolar worlds. Astrobiology 7:252-274.

Kim, H., Smith, H., Mathis, C., Raymond, J., and Walker, S. (2018) Universal scaling across biochemical networks on Earth. bioRxiv doi:10.1101/212118.

Krissansen-Totton, J., Bergsman, D., and Catling, D.C. (2016) On detecting biospheres from chemical thermodynamic disequilibrium in planetary atmospheres. Astrobiology 16:3967.

Krissansen-Totton, J., Olson, S., and Catling, D.C. (2018) Disequilibrium biosignatures over Earth history and implications for detecting exoplanet life. Sci Adv 4, doi:10.1126/sciadv .aao5747.

Lovelock, J.E. (1965) A physical basis for life detection experiments. Nature 207:568-570.

Luger, R. and Barnes, R. (2015) Extreme water loss and abiotic $\mathrm{O}_{2}$ buildup on planets throughout the habitable zones of $\mathrm{M}$ dwarfs. Astrobiology 15:119-143.

Lyons, T.W., Reinhard, C.T., and Planavsky, N.J. (2014) The rise of oxygen in Earth's early ocean and atmosphere. Nature 506:307-315.

Marshall, S.M., Murray, A.R.G., and Cronin, L. (2017) A probabilistic framework for identifying biosignatures using Pathway Complexity. Philos Trans A Math Phys Eng Sci 375, doi:10.1098/rsta.2016.0342.

McKay, C.P. (2011) The search for life in our Solar System and the implications for science and society. Phil Trans Roy Soc A Math Phys Eng Sci 369:594-606.

Meadows, V.S., Reinhard, C.T., Arney, G.N., Parenteau, M.N., Schwieterman, E.W., Domagal-Goldman, S., Lincowski, A.P., Stapelfeldt, K.R., Rauer, H., DasSarma, S., Hegde, S., Narita, N., Deitrick, R., Lustig-Yaeger, J., Lyons, T.W., Siegler, N., and Grenfell, J.L. (2018) Exoplanet biosignatures: understanding oxygen as a biosignature in the context of its environment. Astrobiology 18: 630-662.

Miyashita, H., Adachi, K., Kurano, N., Ikemoto, H., Chihara, M., and Miyachi, S. (1997) Pigment composition of a novel oxygenic photosynthetic prokaryote containing chlorophyll $d$ as the major chlorophyll. Plant Cell Physiol 38: 274-281.

Montanes Rodriguez, P., Pallé, E., Goode, P.R., Hickey, J., Qiu, J., Yurchyshyn, V., Chu, M.C., Kolbe, E., Brown, C.T., and Koonin, S.E. (2004) The Earthshine spectrum. Adv Space Res 34:293-296.

Narita, N., Enomoto, T., Masaoka, S., and Kusakabe, N. (2015) Titania may produce abiotic oxygen atmospheres on habitable exoplanets. Sci Rep 5, doi:10.1038/srep13977.

NASA. (1998) NASA Astrobiology Roadmap, edited by M. Voytek, National Aeronautics and Space Administration, Washington, DC. Available online at https://nai.nasa.gov/ media/roadmap/1998

NASA. (2003) NASA Astrobiology Roadmap, edited by M. Voytek, National Aeronautics and Space Administration, Washington, DC. Available online at https://nai.nasa.gov/ media/roadmap/2003

NASA/JPL-CalTech. (1990) PIA00452: Solar System portraitEarth as 'Pale Blue Dot.' In Voyager Online Data Volumes, Jet Propulsion Laboratory, California Institute of Technology, Pa- sadena, CA. Available online at https://photojournal.jpl.nasa.gov/ catalog/PIA00452

Parviainen, H. (2017) Bayesian methods for exoplanet science. In Handbook of Exoplanets, edited by H.J. Deeg and J.A. Belmontes, Springer International Publishing, Cham, Switzerland, pp 1-24.

Reinhard, C.T., Olson, S.L., Schwieterman, E.W., and Lyons, T.W. (2017) False negatives for remote life detection on ocean-bearing planets: lessons from the early Earth. Astrobiology 17:287-297.

Robinson, T.D., Meadows, V.S., Crisp, D., Deming, D., A'hearn, M.F., Charbonneau, D., Livengood, T.A., Seager, S., Barry, R.K., Hearty, T., Hewagama, T., Lisse, C.M., McFadden, L.A., and Wellnitz, D.D. (2011) Earth as an extrasolar planet: Earth model validation using EPOXI Earth observations. Astrobiology 11:393-408.

Sagan, C. (1994) Pale Blue Dot: A Vision of the Human Future in Space, Random House, New York.

Sagan, C., Thompson, W.R., Carlson, R., Gurnett, D., and Hord, C. (1993) A search for life on Earth from the Galileo spacecraft. Nature 365:715-721.

Sanromá, E., Pallé, E., Parenteau, M.N., Kiang, N.Y., GutíerrezNavarro, A.M., Lopez, R., and Montañes-Rodríguez, P. (2014) Characterizing the purple Earth: modelling the globally integrated spectral variability of the Archean Earth. Astrophys $J$ 780, doi:10.1088/0004-637X/780/1/52.

Schwieterman, E.W., Cockell, C.S., and Meadows, V.S. (2015a) Nonphotosynthetic pigments as potential biosignatures. Astrobiology 15:341-361.

Schwieterman, E.W., Robinson, T.D., Meadows, V.S., Misra, A., and Domagal-Goldman, S. (2015b) Detecting and constraining $\mathrm{N}_{2}$ abundances in planetary atmospheres using collisional pairs. Astrophys $J$ 810, doi:10.1088/0004-637X/ $810 / 1 / 57$.

Schwieterman, E.W., Meadows, V.S., Domagal-Goldman, S.D., Deming, L.D., Arney, G.N., Luger, R., Harman, C.E., Misra, A., and Barnes, R. (2016) Identifying planetary biosignature impostors: spectral features of $\mathrm{CO}$ and $\mathrm{O}_{4}$ resulting from abiotic $\mathrm{O}_{2} / \mathrm{O}_{3}$ production. Astrophys $J$ 819:L13.

Schwieterman, E.W., Kiang, N.Y., Parenteau, M.N., Harman, C.E., DasSarma, S., Fisher, T.M., Arney, G.N., Hartnett, H.E., Reinhard, C.T., Olson, S.L., Meadows, V.S., Cockell, C.S., Walker, S.I., Grenfell, J.L., Hegde, S., Rugheimer, S., $\mathrm{Hu}, \mathrm{R}$., and Lyons, T.W. (2018) Exoplanet biosignatures: a review of remotely detectable signs of life. Astrobiology 18 : 663-708.

Seager, S., Bains, W., and Hu, R. (2013) A biomass-based model to estimate the plausibility of exoplanet biosignature gases. Astrophys J 775, doi:10.1088/0004-637X/775/2/104.

Seager, S., Bains, W., and Petkowski, J.J. (2016) Toward a list of molecules as potential biosignature gases for the search for life on exoplanets and applications to terrestrial biochemistry. Astrobiology 16:465-485.

Segura, A., Kasting, J.F., Meadows, V., Cohen, M., Scalo, J., Crisp, D., Butler, R.A.H., and Tinetti, G. (2005) Biosignatures from Earth-like planets around M dwarfs. Astrobiology 5:706725.

Sparks, W., Hough, J.H., Germer, T.A., Robb, F., and Kolokolova, L. (2012) Remote sensing of chiral signatures on Mars. Planet Space Sci 72:111-115.

Takizawa, K., Minagawa, J., Tamura, M., Kusakabe, N., and Narita, N. (2017) Red-edge position of habitable exopla- 
nets around M-dwarfs. Sci Rep 7, doi:10.1038/s41598-01707948-5.

Tian, F., France, K., Linsky, J.L., Mauas, P.J.D., and Vieytes, M.C. (2014) High stellar FUV/NUV ratio and oxygen contents in the atmospheres of potentially habitable planets. Earth Planet Sci Lett 385:22-27.

Tinetti, G., Rashby, S., and Yung, Y.L. (2006) Detectability of red-edge shifted vegetation on M-star terrestrial planets. Astrophys J 644:L129-L132.

Walker, S.I., Bains, W., Cronin, L., DasSarma, S., Danielache, S., Domagal-Goldman, S., Kacar, B., Kiang, N.Y., Lenardic, A., Reinhard, C.T., Moore, W., Schwieterman, E.W., Shkolnik, E.L., and Smith, H.B. (2018) Exoplanet biosignatures: future directions. Astrobiology 18:779824.

Wordsworth, R. and Pierrehumbert, R. (2014) Abiotic oxygendominated atmospheres on terrestrial habitable zone planets. Astrophys J 785:L20.

Zahnle, K., Haberle, R.M., Catling, D.C., and Kasting, J.F. (2008) Photochemical instability of the ancient martian atmosphere. J Geophys Res: Planets 113, doi:10.1029/ 2008JE003160.
Address correspondence to:

Nancy Y. Kiang

NASA Goddard Institute for Space Studies 2880 Broadway New York, NY 10025

E-mail: Nancy.Y.Kiang@nasa.gov

Submitted 13 March 2018

Accepted 23 March 2018

\section{Abbreviations Used}

EBWWW $=$ Exoplanet Biosignatures Workshop Without Walls

ELTs $=$ Extremely Large Telescopes

GCMs $=$ general circulation models

HabEx $=$ Habitable Exoplanet Observatory

JWST $=$ James Webb Space Telescope

LUVOIR = Large UV/Optical/IR Surveyor

$\mathrm{NExSS}=$ Nexus for Exoplanet System Science $\mathrm{SOC}=$ Scientific Organizing Committee 